\title{
Partial wave decomposition on the lattice and its applications to the HAL QCD method
}

\author{
Takaya Miyamoto, ${ }^{1,2, *}$ Yutaro Akahoshi, ${ }^{1,2}$ Sinya Aoki, ${ }^{1,2}$ Tatsumi Aoyama, ${ }^{1,3,4}$ Takumi Doi, ${ }^{2,5}$ \\ Shinya Gongyo, ${ }^{2}$ and Kenji Sasaki ${ }^{1,2}$ \\ ${ }^{1}$ Center for Gravitational Physics, Yukawa Institute for Theoretical Physics, Kyoto University, \\ Kyoto 606-8502, Japan \\ ${ }^{2}$ RIKEN Nishina Center (RNC), Saitama 351-0198, Japan \\ ${ }^{3}$ Institute of Particle and Nuclear Studies, High Energy Accelerator Research Organization (KEK), \\ Tsukuba, Ibaraki 305-0801, Japan \\ ${ }^{4}$ Kobayashi-Maskawa Institute for the Origin of Particles and the Universe (KMI), Nagoya University, \\ Nagoya 464-8602, Japan \\ ${ }^{5}$ RIKEN Interdisciplinary Theoretical and Mathematical Sciences Program (iTHEMS), \\ Saitama 351-0198, Japan
}

(Received 20 June 2019; accepted 1 April 2020; published 22 April 2020)

\begin{abstract}
The approximated partial-wave decomposition method for discrete data on a cubic lattice, developed by Misner, is applied to the calculation of $S$-wave hadron-hadron scatterings by the HAL QCD method in lattice QCD. We consider the Nambu-Bethe-Salpeter (NBS) wave function for the spin-singlet $\Lambda_{c} N$ system calculated in $(2+1)$-flavor QCD on a $(32 a \mathrm{fm})^{3}$ lattice with lattice spacing $a \simeq 0.0907 \mathrm{fm}$ and $m_{\pi} \simeq 700 \mathrm{MeV}$. We find that by Misner's method the $l=0$ component can be successfully extracted from the NBS wave function projected to the $A_{1}^{+}$representation of the cubic group, which contains small $l \geq 4$ components. Furthermore, while the higher partial-wave components are enhanced so as to produce significant comb-like structures in the conventional HAL QCD potential if the Laplacian approximated by the usual second-order difference is applied to the NBS wave function, such structures are found to be absent in the potential extracted by Misner's method, where the Laplacian can be evaluated analytically for each partial-wave component. Despite the difference in the potentials, the two methods give almost identical results on the central values and magnitude of statistical errors for the fits of the potentials, and consequently on the scattering phase shifts. This indicates not only that Misner's method works well in lattice QCD with the HAL QCD method, but also that the contaminations from higher partial waves in the study of $S$-wave scatterings are well under control even in the conventional HAL QCD method. It will be of interest to study interactions in higher partial-wave channels in the HAL QCD method with Misner's decomposition, where the utility of this new technique may become clearer.
\end{abstract}

DOI: 10.1103/PhysRevD.101.074514

\section{INTRODUCTION}

A determination of hadron-hadron interactions from first principles is one of the ultimate goals in both particle and nuclear physics. In a lattice QCD calculation of a twohadron system, the quantum numbers of the system are specified by the source and/or sink operators in the corresponding correlation function. Among quantum numbers, the partial wave can be specified by the angular

\footnotetext{
*miyamoto@ribf.riken.jp
}

Published by the American Physical Society under the terms of the Creative Commons Attribution 4.0 International license. Further distribution of this work must maintain attribution to the author(s) and the published article's title, journal citation, and DOI. Funded by SCOAP. dependence in terms of the relative coordinate of two hadron operators, in principle. In lattice QCD, however, the extraction of the designated partial wave becomes nontrivial, because the rotational symmetry is broken to the cubic symmetry due to the finite volume (IR effect) as well as the finite lattice spacing (UV effect), which introduces the mixing between different partial waves [1,2]. In addition, a full account of the angular dependence cannot be obtained since the data are only available for discretized spacial coordinates.

In Ref. [3], a general method to (approximately) obtain a radial function in a particular partial wave on the cubic lattice was proposed by Misner, and it has been applied in analyses of gravitational waves simulated using grid points [4]. Applying this method to lattice QCD enables us to verify how much the partial-wave mixing is induced by the 
breaking of the rotational symmetry and evaluate the corresponding systematics in the calculations of hadronhadron interactions. In addition, this method could open a new possibility for extracting interactions in higher partial waves, which are otherwise difficult to study in lattice QCD calculations.

The HAL QCD method is a promising method to calculate hadron-hadron interactions in lattice QCD, in which we construct the hadron-hadron "potential" from the Nambu-Bethe-Salpeter (NBS) wave function and the physical observables such as scattering phase shifts are calculated by solving the Schrödinger equation with the potential in an infinite volume [5-8]. One of the unique features of the HAL QCD method is that the spatial correlation of the NBS wave function is calculated at all discrete points on a cubic lattice, and thus the hadronhadron interactions from the HAL QCD method are expected to be a good application of Misner's method (or Misner's decomposition/extraction). In fact, noticing that not all discrete points at a given radial coordinate $r$ are necessarily transformed to each other by a cubic rotation, it is realized that there is room to develop a better methodology for the partial-wave decomposition than the standard projection method based on the irreducible representation of the cubic group.

In this paper, we apply Misner's method for the first time to the lattice QCD study of hadron-hadron scatterings in the framework of the HAL QCD method. We extract the potential from the $l=0$ ( $S$-wave) component of the NBS wave function using Misner's method, which is then compared with the conventional HAL QCD potential.

In the conventional method, the $S$-wave projection of the NBS wave function is approximated on the lattice by the $A_{1}^{+}$projection as

$$
\psi^{A_{1}^{+}}(\vec{x}) \equiv P^{A_{1}^{+}} \psi(\vec{x})=\frac{1}{48} \sum_{g \in O_{h}} \psi\left(g^{-1} \vec{x}\right),
$$

where the cubic group $O_{h}$ consists of cubic rotations and parity. The $A_{1}^{+}$representation contains not only the $l=0$ component but also higher partial waves with $l \geq 4$. If $l \geq 4$ components of the NBS wave function were absent, the NBS wave function (and the potential) would be isotropic and thus depend only on the radial coordinate $r=|\vec{x}|$. However, we often observe comb-like structures in the potential in terms of $r$ (see, for example, Fig. 2 in Ref. [9]), which represent the anisotropy of the potential. This observation indicates the existence of non-negligible $l \geq 4$ components. We often observe that these comb-like structures lead to superficial fluctuations, whose magnitudes are larger than those of the genuine statistical fluctuations.

Throughout this paper, we write the NBS wave function as $\psi(\vec{x})$, with $\vec{x}=(x, y, z)$ or $(r, \theta, \phi)$, which is expanded in terms of the spherical harmonics $Y_{l m}(\theta, \phi)$ as

$$
\psi(\vec{x})=\sum_{l=0}^{\infty} \sum_{m=-l}^{l} g_{l m}(r) Y_{l m}(\theta, \phi),
$$

where we call $g_{l m}(r)$ the "spherical harmonics amplitude" for a $(l, m)$ component.

This paper is organized as follows. After briefly reviewing the HAL QCD method in Sec. II, we explain Misner's method in detail in Sec. III, together with some remarks on its application to the HAL QCD method. Our main results are given in Sec. IV, where we consider the spin-singlet $\Lambda_{c} N$ system as a representative example. In Sec. IV A, we extract the $l=0$ component of the NBS wave function by Misner's method, where the comb-like structures indeed disappear. However, we find that contaminations from $l \geq 4$ partial waves are small in the $A_{1}^{+}$-projected NBS wave function. In Sec. IV B, we analyze the Laplacian of the NBS wave function by Misner's method. We find that $l \geq 4$ components are enhanced by applying the Laplacian to the NBS wave function in the conventional HAL QCD method, while such a problem is absent in Misner's method, where the Laplacian is calculated analytically for each partial-wave component. In Sec. IV C, we investigate parameter dependencies of the potential in Misner's method. In Sec. V, we calculate the scattering phases shifts from the HAL QCD potentials with the conventional $A_{1}^{+}$ projection and Misner's $S$-wave extraction. We find that both the central values and statistical errors agree in both cases. We briefly discuss a reason for this agreement. A summary and conclusions are presented in Sec. VI. In the Appendix, a simpler but less general method is considered to extract the $l=0$ component from the $A_{1}^{+}$-projected NBS wave function.

\section{HAL QCD METHOD}

In the HAL QCD method [5-8], a nonlocal but energyindependent potential is defined through the Schrödinger equation as

$$
\begin{aligned}
\left(E_{k}-H_{0}\right) \psi^{\left(W_{k}\right)}(\vec{x})= & \int d^{3} x^{\prime} U\left(\vec{x}, \overrightarrow{x^{\prime}}\right) \psi^{\left(W_{k}\right)}\left(\overrightarrow{x^{\prime}}\right), \\
& \left(E_{k}=\frac{|\vec{k}|^{2}}{2 \mu}, H_{0}=-\frac{\vec{\nabla}^{2}}{2 \mu}\right),
\end{aligned}
$$

where the NBS wave function in the center-of-mass frame is given by

$$
\begin{aligned}
& \psi^{\left(W_{k}\right)}(\vec{x}) e^{-W_{k} t} \\
& =\frac{1}{\sqrt{Z_{1}} \sqrt{Z_{2}}} \sum_{\vec{y}}\left\langle 0\left|B_{1}(\vec{x}+\vec{y}, t) B_{2}(\vec{y}, t)\right| 2 B ; W_{k}\right\rangle .
\end{aligned}
$$

Here we consider a two-baryon system as a representative case, where $B_{i}(\vec{x}, t)(i=1,2)$ is the local interpolating operator for a baryon $B_{i}$ with renormalization factor $\sqrt{Z_{i}}$. 
The state $\left|2 B ; W_{k}\right\rangle$ stands for a QCD eigenstate for the two-baryon system with total energy $W_{k}=\sqrt{|\vec{k}|^{2}+m_{B_{1}}^{2}}+$ $\sqrt{|\vec{k}|^{2}+m_{B_{2}}^{2}}$, with baryon masses $m_{B_{1,2}}$ and relative momentum $\vec{k}$, and $\mu$ denotes the reduced mass. Since the asymptotic behavior of the NBS wave function at large $r=|\vec{x}|$ is identical to that of the scattering wave in quantum mechanics, whose phase shift is the phase of the QCD $S$ matrix $[10,11]$, the potential defined from the NBS wave functions reproduces the scattering phase shifts in QCD. Note that the nonlocal potential is constructed so as to be energy independent below the inelastic threshold $[6,8]$.

In terms of the NBS wave functions, the two-baryon four-point correlation function is expressed as

$$
\begin{aligned}
G\left(\vec{x}, t-t_{0}\right)= & \sum_{\vec{y}}\left\langle 0\left|B_{1}(\vec{x}+\vec{y}, t) B_{2}(\vec{y}, t) \mathcal{J}^{\left(J^{P}\right)}\left(t_{0}\right)\right| 0\right\rangle \\
= & \sum_{n} \sum_{\vec{y}}\left\langle 0\left|B_{1}(\vec{x}+\vec{y}, t) B_{2}(\vec{y}, t)\right| 2 B ; W_{n}\right\rangle \\
& \times\left\langle 2 B ; W_{n}\left|\mathcal{J}^{\left(J^{P}\right)}\left(t_{0}\right)\right| 0\right\rangle+\cdots \\
= & \sqrt{Z_{1}} \sqrt{Z_{2}} \sum_{n} \psi^{\left(W_{n}\right)}(\vec{x}) e^{-W_{n}\left(t-t_{0}\right)} A_{n}+\cdots, \\
A_{n} \equiv & \left\langle 2 B ; W_{n}\left|\mathcal{J}^{\left(J^{P}\right)}(0)\right| 0\right\rangle,
\end{aligned}
$$

where $\mathcal{J}^{\left(J^{P}\right)}\left(t_{0}\right)$ stands for a source operator defined so as to create two-baryon states at $t=t_{0}$ with total angular momentum $J$ and parity $P$, and the ellipsis represents contributions from inelastic states. For a sufficiently large $t-t_{0}$, the NBS wave function for the ground state is extracted from the four-point function as

$$
\begin{aligned}
G\left(\vec{x}, t-t_{0}\right) \rightarrow & \sqrt{Z_{1}} \sqrt{Z_{2}} \psi^{\left(W_{0}\right)}(\vec{x}) e^{-W_{0}\left(t-t_{0}\right)} A_{0} \\
& +\mathcal{O}\left(e^{-W_{1}\left(t-t_{0}\right)}\right) .
\end{aligned}
$$

In practice, however, this extraction of the NBS wave function from the ground-state saturation is very difficult due to increasing statistical noise at large $t-t_{0}$ [12-14]. Therefore, in Ref. [7] an improved method was proposed to extract the potential without the requirement of the groundstate saturation. We consider the normalized baryon fourpoint correlation function ( $R$ correlator) defined by

$$
\begin{aligned}
R\left(\vec{x}, t-t_{0}\right) & \equiv \frac{G\left(\vec{x}, t-t_{0}\right)}{e^{-m_{B_{1}}\left(t-t_{0}\right)} e^{-m_{B_{2}}\left(t-t_{0}\right)}} \\
& =\sqrt{Z_{1} Z_{2} \sum_{n} \psi^{\left(W_{n}\right)}(\vec{x}) e^{-\Delta W_{n}\left(t-t_{0}\right)} A_{n}+\cdots,}
\end{aligned}
$$

where $\Delta W_{n}=W_{n}-\left(m_{B_{1}}+m_{B_{2}}\right)$. If contributions from inelastic states are negligible ("elastic state saturation"), this $R$ correlator satisfies

$$
\begin{aligned}
& {\left[\left(\frac{1+3 \delta^{2}}{8 \mu}\right) \frac{\partial^{2}}{\partial t^{2}}-\frac{\partial}{\partial t}-H_{0}+\mathcal{O}\left(\frac{\delta^{2}}{2 m_{B_{1}} m_{B_{2}}} \frac{\partial^{3}}{\partial t^{3}}\right)\right] R\left(\vec{x}, t-t_{0}\right)} \\
& \quad=\int d^{3} x^{\prime} U\left(\vec{x}, \overrightarrow{x^{\prime}}\right) R\left(\overrightarrow{x^{\prime}}, t-t_{0}\right)
\end{aligned}
$$

where $\delta=\left(m_{B_{1}}-m_{B_{2}}\right) /\left(m_{B_{1}}+m_{B_{2}}\right)$. Since the elasticstate saturation can generally be achieved at much smaller $t-t_{0}$ than for ground-state saturation, we can obtain reliable results from this "time-dependent HAL QCD method" [12-15].

In order to handle the nonlocality of the potential, we introduce the derivative expansion as

$$
U\left(\vec{x}, \overrightarrow{x^{\prime}}\right)=V(\vec{x}, \vec{\nabla}) \delta^{(3)}\left(\vec{x}-\overrightarrow{x^{\prime}}\right),
$$

where $V(\vec{x}, \vec{\nabla})$ is expanded in terms of $\vec{\nabla}$ [16]. At low energies, since the leading-order (LO) potential of the derivative expansion dominates [15], the interaction for the $S$-wave spin-singlet system is well approximated by the LO central potential, given as

$$
V_{\mathrm{LO}}^{1} S_{0}(r)=\frac{\left[\left(\frac{1+3 \delta^{2}}{8 \mu}\right) \frac{\partial^{2}}{\partial t^{2}}-\frac{\partial}{\partial t}-H_{0}\right] R^{1} S_{0}\left(\vec{x}, t-t_{0}\right)}{R^{1} S_{0}\left(\vec{x}, t-t_{0}\right)},
$$

where the $R$ correlator for the ${ }^{1} S_{0}$ state is defined as

$$
R^{1} S_{0}\left(\vec{x}, t-t_{0}\right) \equiv P^{A_{1}^{+}} P^{S=0} R\left(\vec{x}, t-t_{0}\right) .
$$

Here $P^{S=0}$ represents the projection to the state with total spin $S=0$, while $P^{A_{1}^{+}}$stands for the projection to the $A_{1}^{+}$ representation in Eq. (1). As we have explained before, the $A_{1}^{+}$projection contains not only a $l=0$ component but also $l \geq 4$ components, which produce angular dependencies for the $R$ correlator as well as the potential.

\section{APPROXIMATED PARTIAL-WAVE DECOMPOSITION}

\section{A. Misner's method}

Let us consider the extraction $g_{l m}(r)$ for a given $r \equiv$ $|\vec{x}|=R$ from the NBS wave function. In the continuum space, we can obtain $g_{l m}(R)$ by taking the spherical surface integral at $r=R$ as

$$
g_{l m}(R)=\int_{S} d \Omega \overline{Y_{l m}(\theta, \phi)} \psi(\vec{x} ; r=R),
$$

because of the orthogonality of the spherical harmonics,

$$
\int_{S} d \Omega \overline{Y_{l m}(\theta, \phi)} Y_{l^{\prime} m^{\prime}}(\theta, \phi)=\delta_{l l^{\prime}} \delta_{m m^{\prime}}
$$

where the overline represents complex conjugation. This is even true in a finite $L_{s}^{3}$ box in the continuum space for $0<R \leq L_{s} / 2$. 
In a discrete space such as the cubic lattice, however, we can not obtain $g_{l m}(R)$ for any $R$ since $N_{R}$ (the number of points that satisfy $r=R$ ) is finite, so the infinitedimensional matrix

$$
\begin{aligned}
\mathcal{G} & \equiv\left\{\mathcal{G}_{l m, l^{\prime} m^{\prime}}\right. \\
& \left.\equiv \frac{1}{N_{R}} \sum_{\vec{x} \in\{\vec{x} \mid r=R\}} \overline{Y_{l m}(\theta, \phi)} Y_{l^{\prime} m^{\prime}}(\theta, \phi)|| m|\leq l,| m^{\prime} \mid \leq l^{\prime}\right\}
\end{aligned}
$$

is noninvertible due to its zero eigenvalues. If one knows that contributions from higher partial waves are negligible, one can introduce some approximation in order to obtain $g_{l m}(R)$ for small $l$ and some restricted $R$. For example, we may impose the condition that $l, l^{\prime} \leq l_{\max }$, where $l_{\max }$ is chosen so that the finite-dimensional matrix $\mathcal{G}^{l_{\max }} \equiv$ $\left\{\mathcal{G}_{l m, l^{\prime} m^{\prime}} \mid l, l^{\prime} \leq l_{\max }\right\}$ becomes invertible. In the Appendix we consider this approximation in detail.

In Ref. [3], a more general and sophisticated approximation was proposed. To explain the method, let us start from the continuum case. We first introduce the basis functions in the radial coordinate $G_{n}^{R, \Delta}(r)$ $(n=0, \ldots, \infty)$ which are orthonormal in the radial interval $[R-\Delta, R+\Delta]$,

$$
\int_{R-\Delta}^{R+\Delta} d r r^{2} \overline{G_{n}^{R, \Delta}(r)} G_{m}^{R, \Delta}(r)=\delta_{n m} .
$$

One of the candidates for $G_{n}^{R, \Delta}(r)$ is given by

$$
G_{n}^{R, \Delta}(r)=\frac{1}{r} \sqrt{\frac{2 n+1}{2 \Delta}} P_{n}\left(\frac{r-R}{\Delta}\right),
$$

where $P_{n}(r)$ is the Legendre polynomial, which obviously satisfies Eq. (15). When we consider a spherical shell $S_{R, \Delta}$ with thickness $2 \Delta$ surrounding the spherical surface $r=R$ defined by

$$
S_{R, \Delta} \equiv\{\vec{x} \mid R-\Delta \leq r \leq R+\Delta\},
$$

an orthonormal basis function $\mathcal{Y}_{n l m}^{R, \Delta}(r, \theta, \phi) \equiv$ $G_{n}^{R, \Delta}(r) Y_{l m}(\theta, \phi)$ obeys

$$
\int_{S_{R, \Delta}} d^{3} x \overline{\mathcal{Y}_{n l m}^{R, \Delta}(r, \theta, \phi)} \mathcal{Y}_{n^{\prime} l^{\prime} m^{\prime}}^{R, \Delta}(r, \theta, \phi)=\delta_{n n^{\prime}} \delta_{l l^{\prime}} \delta_{m m^{\prime}},
$$

where the integral over $S_{R, \Delta}$ is defined as

$$
\int_{S_{R, \Delta}} d^{3} x \equiv \int_{R-\Delta}^{R+\Delta} r^{2} d r \int_{S} d \Omega
$$

The NBS wave function in a spherical shell $S_{R, \Delta}$ is expanded in terms of the orthonormal basis functions as

$$
\psi(\vec{x})=\sum_{n=0}^{\infty} \sum_{l=0}^{\infty} \sum_{m=-l}^{l} c_{n l m}^{R, \Delta} \mathcal{Y}_{n l m}^{R, \Delta}(r, \theta, \phi)
$$

with coefficients $c_{n l m}^{R, \Delta}$, which can be determined by

$$
c_{n l m}^{R, \Delta}=\int_{S_{R, \Delta}} d^{3} x \overline{\mathcal{Y}_{n l m}^{R, \Delta}(r, \theta, \phi)} \psi(\vec{x}) .
$$

We finally obtain the spherical harmonics amplitude $g_{l m}(r)$ for $R-\Delta \leq r \leq R+\Delta$ as

$$
g_{l m}(r)=\sum_{n=0}^{\infty} c_{n l m}^{R, \Delta} G_{n}^{R, \Delta}(r) .
$$

Reference [3] employed $\mathcal{Y}_{n l m}^{R, \Delta}(r, \theta, \phi)$ as the basis function for the approximation in the case of the discrete space on a cubic lattice. In this case, the volume integral is replaced by a discrete sum as

$$
\int_{S_{R, \Delta}} d^{3} x \Rightarrow \sum_{\vec{x}} \omega^{R, \Delta}(\vec{x}),
$$

where $\omega^{R, \Delta}(\vec{x})$ is a weight factor, which corresponds to a volume of the overlapped region between the shell $S_{R, \Delta}$ and a unit cube around the point $\vec{x}$. For example, if the unit cube lies entirely inside the shell $S_{R, \Delta}, \omega^{R, \Delta}(\vec{x})=a^{3}$ with lattice spacing $a$, while $\omega^{R, \Delta}(\vec{x})=0$ when the unit cube lies entirely outside the shell. Since $\omega^{R, \Delta}(\vec{x})$ in the general cases is rather complicated, it was approximated in Ref. [3] as

$\omega^{R, \Delta}(\vec{x})= \begin{cases}a^{3} & \text { for }|r-R|<\Delta-\frac{1}{2} a, \\ 0 & \text { for }|r-R|>\Delta+\frac{1}{2} a, \\ a^{2}\left(\Delta+\frac{1}{2} a-|R-r|\right) & \text { otherwise, }\end{cases}$

which corresponds to the overlapped volume of a unit cube parallel to the radial direction. Using this, we define the inner product of functions $f(\vec{x})$ and $g(\vec{x})$ in the shell $S_{R, \Delta}$ as

$$
\langle f \mid g\rangle_{S_{R, \Delta}} \equiv \sum_{\vec{x}} \omega^{R, \Delta}(\vec{x}) \overline{f(\vec{x})} g(\vec{x}) .
$$

Let us consider $\mathcal{G}_{A A^{\prime}} \equiv\left\langle\mathcal{Y}_{A}^{R, \Delta} \mid \mathcal{Y}_{A^{\prime}}^{R, \Delta}\right\rangle_{S_{R, \Delta}}$, with the shorthand notation $A=(n, l, m)$. The finite-dimensional Hermitian matrix $\mathcal{G}$ constructed from $\mathcal{G}_{A A^{\prime}}$ with the restriction that $n, n^{\prime} \leq n_{\max }$ and $l, l^{\prime} \leq l_{\max }$ becomes invertible if one properly chooses $n_{\max }$ and $l_{\max }$. Using $\mathcal{G}$ (whose dependencies on $n_{\max }$ and $l_{\max }$ are implicit here), one can define the dual basis functions $\tilde{\mathcal{Y}}_{A}^{R, \Delta}$ as

$$
\tilde{\mathcal{Y}}_{A}^{R, \Delta}(\vec{x}) \equiv \sum_{B}^{\prime} \mathcal{Y}_{B}^{R, \Delta}(\vec{x}) \mathcal{G}_{B A}^{-1},
$$


which satisfies

$$
\begin{aligned}
\left\langle\tilde{\mathcal{Y}}_{A}^{R, \Delta} \mid \mathcal{Y}_{B}^{R, \Delta}\right\rangle_{S_{R, \Delta}} & =\sum_{C}^{\prime} \mathcal{G}_{A C}^{-1}\left\langle\mathcal{Y}_{C}^{R, \Delta} \mid \mathcal{Y}_{B}^{R, \Delta}\right\rangle_{S_{R, \Delta}} \\
& =\sum_{C}^{\prime} \mathcal{G}_{A C}^{-1} \mathcal{G}_{C B}=\delta_{A B},
\end{aligned}
$$

where the prime in the summation indicates the upper bounds $n_{\max }$ and $l_{\max }$.

Assuming that $c_{n l m}^{R, \Delta}$ is negligibly small for $l>l_{\max }$ or $n>n_{\max }$, Eq. (20) is approximately written as

$$
\psi(\vec{x}) \simeq \sum_{n=0}^{n_{\max }} \sum_{l=0}^{l_{\max }} \sum_{m=-l}^{l} c_{n l m}^{R, \Delta} \mathcal{Y}_{n l m}^{R, \Delta}(r, \theta, \phi)
$$

with the coefficient $c_{n l m}^{R, \Delta}=\left\langle\tilde{\mathcal{Y}}_{n l m}^{R, \Delta} \mid \psi\right\rangle_{S_{R, \Delta}}$. Finally, the spherical harmonics amplitude $g_{l m}(R)$ can be approximated as

$$
g_{l m}(R) \simeq \sum_{n=0}^{n_{\max }} c_{n l m}^{R, \Delta} G_{n}^{R, \Delta}(R) .
$$

\section{B. Misner's method as a minimization}

Misner's method can also be understood from the viewpoint of least-square minimization, and we here give the explicit correspondence following Ref. [17].

$$
\boldsymbol{Y}=\left(\begin{array}{cc}
\mathcal{Y}_{0,0,0}^{R, \Delta}\left(\vec{x}_{1}\right) & \mathcal{Y}_{0,1,-1}^{R, \Delta}\left(\vec{x}_{1}\right) \\
\vdots & \vdots \\
\mathcal{Y}_{0,0,0}^{R, \Delta}\left(\vec{x}_{N_{R, \Delta}}\right) & \mathcal{Y}_{0,1,-1}^{R, \Delta}\left(\vec{x}_{N_{R, \Delta}}\right)
\end{array}\right.
$$$$
\left.\begin{array}{ccc}
\mathcal{Y}_{0,1,0}^{R, \Delta}\left(\vec{x}_{1}\right) & \cdots & \mathcal{Y}_{2,2,2}^{R, \Delta}\left(\vec{x}_{1}\right) \\
\vdots & & \vdots \\
\mathcal{Y}_{0,1,0}^{R, \Delta}\left(\vec{x}_{N_{R, \Delta}}\right) & \cdots & \mathcal{Y}_{2,2,2}^{R, \Delta}\left(\vec{x}_{N_{R, \Delta}}\right)
\end{array}\right),
$$

where the number of columns is $M=27$. Defining a $N_{R, \Delta} \times N_{R, \Delta}$ diagonal matrix $\boldsymbol{W}$ for the nonzero weight $\omega^{R, \Delta}(\vec{x})$, the $M \times M$ matrix $\equiv\left\{\mathcal{G}_{A B}\right\}$ is simply written as $\mathcal{G}=\boldsymbol{Y}^{\dagger} \boldsymbol{W} \boldsymbol{Y}$.

Using these notations, we introduce a trial $N_{R, \Delta^{-}}$ component vector function $\tilde{\Psi} \equiv \boldsymbol{Y} \tilde{\boldsymbol{C}}$ as

$$
\tilde{\Psi}_{i} \equiv \tilde{\psi}\left(\vec{x}_{i}\right)=\sum_{n=0}^{n_{\max }} \sum_{l=0}^{l_{\max }} \sum_{m=-l}^{l} \boldsymbol{Y}_{i, n l m} \tilde{\boldsymbol{C}}_{n l m},
$$

where a $M$-component vector $\tilde{\boldsymbol{C}}$ corresponds to fit parameters that should minimize

$$
F(\tilde{\boldsymbol{C}})=(\tilde{\Psi}-\Psi)^{\dagger} \boldsymbol{W}(\tilde{\boldsymbol{\Psi}}-\Psi)=(\boldsymbol{Y} \tilde{\boldsymbol{C}}-\Psi)^{\dagger} \boldsymbol{W}(\boldsymbol{Y} \tilde{\boldsymbol{C}}-\Psi) .
$$

Since $d F(\tilde{\boldsymbol{C}}) / d \tilde{\boldsymbol{C}}=0$ at the minimum $\tilde{\boldsymbol{C}}=\tilde{\boldsymbol{C}}_{\text {min }}$, we obtain

$$
\tilde{\boldsymbol{C}}_{\min }=\left(\boldsymbol{Y}^{\dagger} \boldsymbol{W} \boldsymbol{Y}\right)^{-1} \boldsymbol{Y}^{\dagger} \boldsymbol{W} \Psi=\mathcal{G}^{-1} \boldsymbol{Y}^{\dagger} \boldsymbol{W} \Psi,
$$

Let us denote a $N_{R, \Delta}$-component vector $\Psi$ of the NBS wave function as

$$
\Psi=\left(\begin{array}{c}
\psi\left(\vec{x}_{1}\right) \\
\psi\left(\vec{x}_{2}\right) \\
\vdots \\
\psi\left(\vec{x}_{N_{R, \Delta}}\right)
\end{array}\right),
$$

where $N_{R, \Delta}$ is the number of points in the shell $S_{R, \Delta}$ or, equivalently, the number of data with nonzero $\omega^{R, \Delta}(\vec{x})$. Similarly, we define a $N_{R, \Delta} \times M$ rectangular matrix $\boldsymbol{Y}$ of the basis functions, whose components are defined by

$$
\begin{aligned}
& \boldsymbol{Y}_{i, n l m}=\mathcal{Y}_{n, l, m}^{R, \Delta}\left(\vec{x}_{i}\right), \quad 1 \leq i \leq N_{R, \Delta}, \\
& 0 \leq n \leq n_{\max }, \quad 0 \leq l \leq l_{\max }, \quad|m| \leq l,
\end{aligned}
$$

where $M$ is the number of basis functions and is given by $M=\left(n_{\max }+1\right)\left(l_{\max }+1\right)^{2}$. For $n_{\max }=2$ and $l_{\max }=2$, for example, $\boldsymbol{Y}$ becomes

so that

$$
\begin{aligned}
\left(\tilde{\Psi}_{\min }\right)_{i} & =\left(\boldsymbol{Y} \tilde{\boldsymbol{C}}_{\min }\right)_{i}=\sum_{A}^{\prime} \mathcal{Y}_{A}^{R, \Delta}\left(\vec{x}_{i}\right)\left\langle\tilde{\mathcal{Y}}_{A}^{R, \Delta} \mid \psi\right\rangle_{S_{R, \Delta}} \\
& =\sum_{n=0}^{n_{\max }} \sum_{l=0}^{l_{\max }} \sum_{m=-l}^{l} \mathcal{Y}_{n l m}^{R, \Delta}\left(\vec{x}_{i}\right) c_{n l m}^{R, \Delta},
\end{aligned}
$$

which agrees with Misner's method [Eq. (28)]. Therefore, Misner's method is equivalent to finding a solution of $\tilde{\psi}\left(\vec{x}_{i}\right)$ that minimizes the difference between the data $\psi\left(\vec{x}_{i}\right)$ and the fit function $\tilde{\psi}\left(\vec{x}_{i}\right)$ defined by the norm $\langle\tilde{\psi}-\psi \mid \tilde{\psi}-\psi\rangle_{S_{R, \Delta}}$.

\section{Remarks}

The calculation of the potential in the HAL QCD method requires applying the Laplacian to the NBS wave function, which is conventionally approximated by a finite difference, and thus contains discretization errors. In the 
application of Misner's decomposition to the HAL QCD method, we can instead employ an analytical expression for the Laplacian, which operates on the (approximately obtained) partial wave $g_{l m}(r) Y_{l m}(\theta, \phi)$ as

$$
\begin{aligned}
\vec{\nabla}^{2} & {\left[g_{l m}(r) Y_{l m}(\theta, \phi)\right] } \\
& =\left\{\frac{1}{r} \frac{\partial^{2}}{\partial r^{2}}\left[r g_{l m}(r)\right]-\frac{l(l+1)}{r^{2}} g_{l m}(r)\right\} Y_{l m}(\theta, \phi),
\end{aligned}
$$

where

$$
\begin{aligned}
\frac{1}{r} \frac{\partial^{2}}{\partial r^{2}}\left[r g_{l m}(r)\right] & =\sum_{n=0}^{n_{\max }} c_{n l m}^{R, \Delta} \frac{1}{r} \frac{\partial^{2}}{\partial r^{2}}\left[r G_{n}^{R, \Delta}(r)\right] \\
& =\frac{1}{r} \sum_{n=0}^{n_{\max }} c_{n l m}^{R, \Delta} \frac{\partial^{2}}{\partial r^{2}}\left[\sqrt{\frac{2 n+1}{2 \Delta}} P_{n}\left(\frac{r-R}{\Delta}\right)\right] \\
& =\frac{1}{r \Delta^{2}} \sum_{n=0}^{n_{\max }} \sqrt{\frac{2 n+1}{2 \Delta}} c_{n l m}^{R, \Delta} P_{n}^{\prime \prime}\left(\frac{r-R}{\Delta}\right),
\end{aligned}
$$

and $P_{n}^{\prime \prime}$ is the second-order derivative for the Legendre polynomial. Unlike the conventional HAL QCD method in which the difference operator for the Laplacian is applied to (all partial-wave components of) the NBS wave function, it is clear that the analytic derivative in Misner's method does not induce contributions from partial waves other than the targeted one. A comparison between the two implementations of the Laplacian operator will be given in Sec. IV B.

In Misner's method, it is practically important to choose $n_{\max }, l_{\max }$, and $\Delta$ appropriately. While larger $n_{\max }, l_{\max }$ and smaller $\Delta$ give a better approximation of the NBS wave function, they lead to a small $N_{R, \Delta}-M$ that may cause some numerical instability due to small eigenvalues of $\mathcal{G}_{A B}$ [18] or may give an overfitting, where $N_{R, \Delta}$ and $M$ correspond to the number of data and fit parameters, respectively. For example, if the variation of the NBS wave function in the radial coordinate is large, one should increase $n_{\max }$ to better approximate the spherical harmonics amplitude $g_{l m}(r)$, but not too much so as to avoid instability or overfitting. The scaling of the discretization error in Misner's method was discussed in Ref. [19]. It was found that the error depends on $\Delta$ as $\mathcal{O}\left(\Delta^{n_{\max }+2}\right)$, which also indicates that the choice of $\Delta=\mathcal{O}(a)$ is preferable. In addition, the volume integral in the shell with the approximated weight [Eq. (24)] gives a $\mathcal{O}\left(a^{2}\right)$ error with the choice $\Delta=\mathcal{O}(a)$. Therefore, the choice of parameters $n_{\max }=2$ with $\Delta=\mathcal{O}(a)$ is found to be good for second-order accuracy. The author [19] also suggested $\Delta=3 a / 4$ as a rule of thumb from numerical investigations, but we have to examine whether the results are stable against changes in the parameters $\Delta, n_{\max }$, and $l_{\max }$ case by case, as will be presented in the next section.

In practice, the most costly calculation in Misner's method is the construction of the matrix $\mathcal{G}_{A B}$. Once we calculate the matrix, however, we can use it for different lattice data (e.g., NBS wave functions calculated on different gauge samples). Therefore, it is better to calculate the dual basis functions [Eq. (26)] once before the calculation of the spherical harmonics amplitude from NBS wave functions and use them in later analyses. One possible obstacle in this procedure is that the dual basis functions $\tilde{\mathcal{Y}}_{n l m}^{R, \Delta}(\vec{x})$ require a large amount of memory to store, $L^{3}\left(n_{\max }+1\right)\left(l_{\max }+1\right)^{2} \times 16$ bytes for each given value of $R$. For example, the required memory for $\tilde{\mathcal{Y}}_{n l m}^{R, \Delta}(\vec{x})$ with $L=32, n_{\max }=4$, and $l_{\max }=6$ becomes $32^{3} \times 5 \times 7^{2} \times$ 16 bytes $=122.5 \mathrm{MB}$. In order to reduce the memory usage by a factor of $\left(n_{\max }+1\right)$, we instead store

$$
F_{l m}^{R, \Delta}(\vec{x}) \equiv \sum_{n=0}^{n_{\max }} \overline{G_{n}^{R, \Delta}(R)} \tilde{\mathcal{Y}}_{n l m}^{R, \Delta}(\vec{x}),
$$

which needs only $24.5 \mathrm{MB}$. Using this function, the spherical harmonics amplitude $g_{l m}(R)$ can be calculated directly as

$$
\begin{aligned}
\left\langle F_{l m}^{R, \Delta} \mid \psi\right\rangle_{S_{R, \Delta}} & =\sum_{n=0}^{n_{\max }} G_{n}^{R, \Delta}(R)\left\langle\tilde{\mathcal{Y}}_{n l m}^{R, \Delta} \mid \psi\right\rangle_{S_{R, \Delta}} \\
& =\sum_{n=0}^{n_{\max }} G_{n}^{R, \Delta}(R) c_{n l m}^{R, \Delta} \simeq g_{l m}(R) .
\end{aligned}
$$

Furthermore, it is sufficient to store $F_{l m}^{R, \Delta}(\vec{x})$ only at a point $\vec{x}$ included in the shell $S_{R, \Delta}$ where the weight function $\omega^{R, \Delta}(\vec{x})$ is nonzero, which leads to a large reduction in memory usage.

\section{HAL QCD POTENTIALS WITH MISNER'S METHOD}

For the numerical calculation, we consider the spinsinglet $\Lambda_{c} N$ system in $(2+1)$-flavor full lattice QCD with the renormalization-group-improved Iwasaki gluon action and a nonperturbatively $\mathcal{O}(a)$-improved Wilson-clover quark action on a $(32 a \mathrm{fm})^{3} \times(64 a \mathrm{fm})$ volume with lattice spacing $a \simeq 0.0907 \mathrm{fm}$ at $m_{\pi} \simeq 700 \mathrm{MeV}$. We apply Misner's method to the same NBS wave function data calculated in Ref. [20], where results using the conventional HAL QCD method were given. We employ a walltype source operator and thus the $\Lambda_{c} N$ system belongs to the $A_{1}^{+}$representation of the cubic group. In order to reduce the statistical fluctuations, we also impose the $A_{1}^{+}$projection on the sink operator as given in Eq. (1). Recall that the $A_{1}^{+}$representation contains the partial waves $l=0,4,6, \ldots$. In this study, we consider the $A_{1}^{+}$-projected $R$ correlator taken at $t-t_{0}=13 a$. There are 399 configurations, and the statistical errors are estimated by the jackknife method with a bin size of 57 configurations (for a total of seven bins). For more details on the simulation setup, see Ref. [20]. 


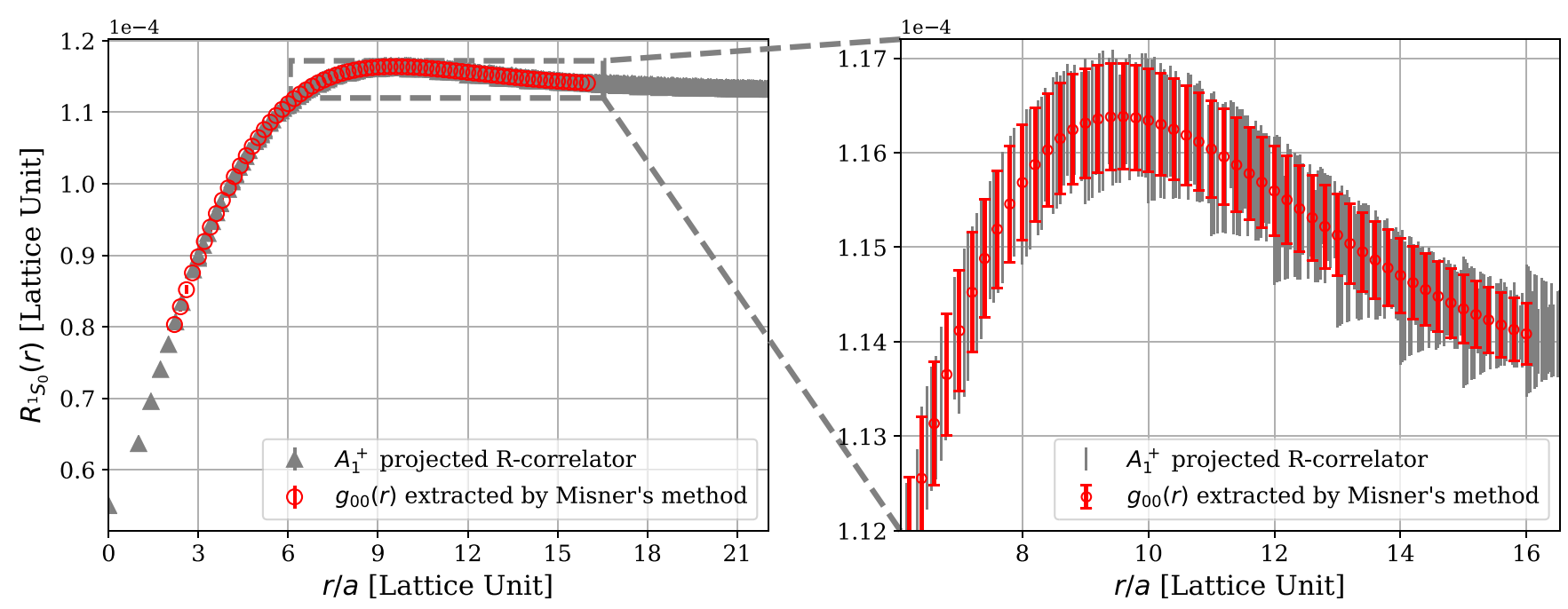

FIG. 1. The $R$ correlator for the spin-singlet $\Lambda_{c} N$ system at $t-t_{0}=13 a$ for $m_{\pi} \simeq 700 \mathrm{MeV}$. The gray points show the $R$ correlator with the $A_{1}^{+}$projection divided by $Y_{00}$, while the red points correspond to the spherical harmonics amplitude $g_{00}(r)$ calculated by Misner's method.

\section{A. NBS wave function for the spin-singlet $\Lambda_{c} N$ system}

Figure 1 shows the results of the $R$ correlator defined in Eq. (7) for the spin-singlet $\Lambda_{c} N$ system. The gray points represent the $A_{1}^{+}$-projected $R$ correlator divided by $Y_{00}$, which was actually used in Ref. [20] to construct the $\Lambda_{c} N$ potential. The red points correspond to the spherical harmonics amplitude $g_{l m}(r)$ for the $l=m=0$ component calculated by Misner's method for the radial coordinate $r$ from $2 a$ to $16 a$ with the interval $\Delta r=0.2 a$, so that some data is used several times. We do not use Misner's method for $r<2 a$ and $r>L_{s} / 2=16 a$ : in the former case, the number of data points in the spherical shell $S_{r, \Delta}$ is too small, whereas in the latter case the spherical shell is not contained in the $L_{s}^{3}$ cubic lattice. Here we employ $\Delta=a$, $n_{\max }=2$, and $l_{\max }=4$ as the parameters in Misner's method, and we find that $g_{00}(r)$ has a weak parameter dependence.

Figure 1 shows small comb-like structures in the $A_{1}^{+}$projected $R$ correlator, which however do not appear in the $l=0$ component extracted by Misner's method. This observation indicates that $l \geq 4$ components exist in the $A_{1}^{+}$-projected $R$ correlator and their angular dependencies become manifest as the comb-like structures in the radial coordinate. Such higher partial-wave components can be explicitly extracted by Misner's method, as shown in Fig. 2 for the $l=4$ component $\left[g_{40}(r)=g_{4 \pm 4}(r)\right]$. Note that $g_{4 m}(r)=0$ for $m \neq 0, \pm 4$ for the $A_{1}^{+}$representation. We find that the $l=4$ component indeed exists, but its magnitude is small [by a factor of $\mathcal{O}\left(10^{-3}\right)$ compared to that of the $l=0$ component]. On the other hand, the

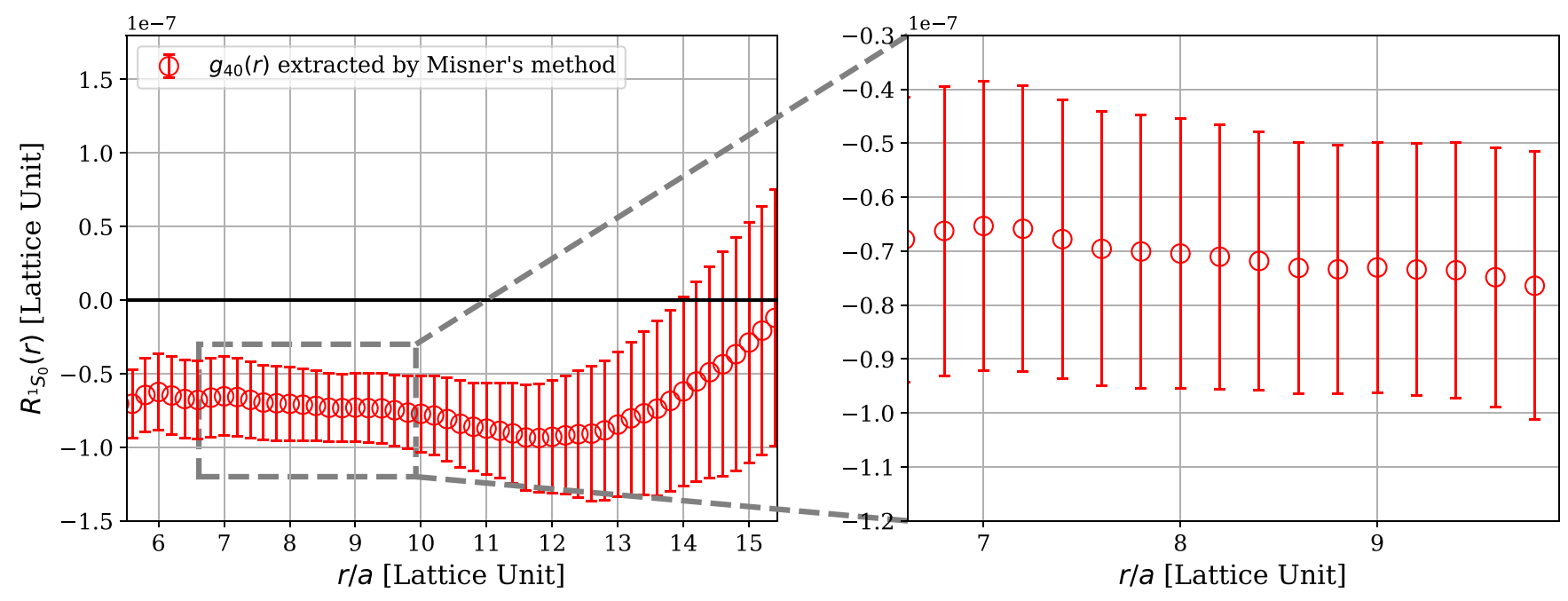

FIG. 2. The spherical harmonics amplitudes for the $l=4$ component extracted by Misner's method. 


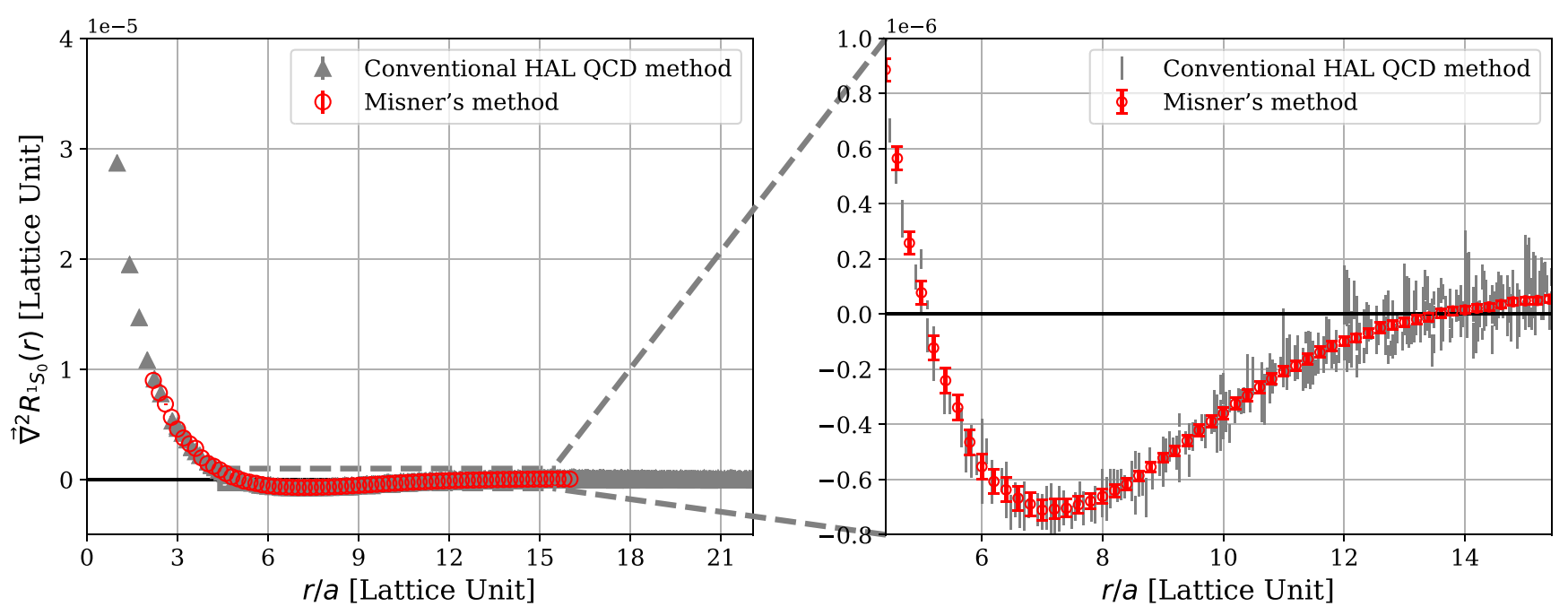

FIG. 3. The Laplacian applied to the $R$ correlator in the conventional method (gray points) and in Misner's method (red points). The Laplacian is defined by a finite second-order difference in the former, while it is analytically calculated using Eq. (35) in the latter.

absence of comb-like structures in the $l=0,4$ components obtained by Misner's method with $l_{\max }=4$ indicates that $l \leq 4$ components are sufficient to explain the $A_{1}^{+}$-projected $R$ correlator, which is explicitly confirmed by observing that the $l=6$ component extracted by Misner's method with $l_{\max }=6$ is actually negligible. The mixing of the $l=4$ component is most likely induced due to the rotational symmetry breaking by the finite volume (IR effect), except for $r \lesssim a$ where there could also exist an effect from the finite lattice spacing (UV effect).

\section{B. Laplacian and HAL QCD potential}

We now study the effect of applying the Laplacian to the NBS wave function. Note that the term containing the Laplacian, i.e., the third term on the rhs of Eq. (10) is known to give the dominant contribution for the potential. In the conventional HAL QCD method, the Laplacian approximated by a finite second-order difference is applied to (all partial-wave components of) the NBS wave function, while it is analytically calculated as Eq. (35) in Misner's method for the designated partial-wave component. In Fig. 3, we compare the Laplacian applied to the NBS wave functions between Misner's method (red points) and the conventional HAL QCD method (gray points). In the case of Misner's method, the Laplacian analytically applied to $g_{00}(r)$ does not exhibit the comb-like structure. In the case of the conventional HAL QCD method, on the other hand, we find that the comb-like structures are much larger than those of the $R$ correlator itself, indicating that the $l \geq 4$ components are larger for the conventional Laplacian. The partial-wave decomposition of the conventional Laplacian
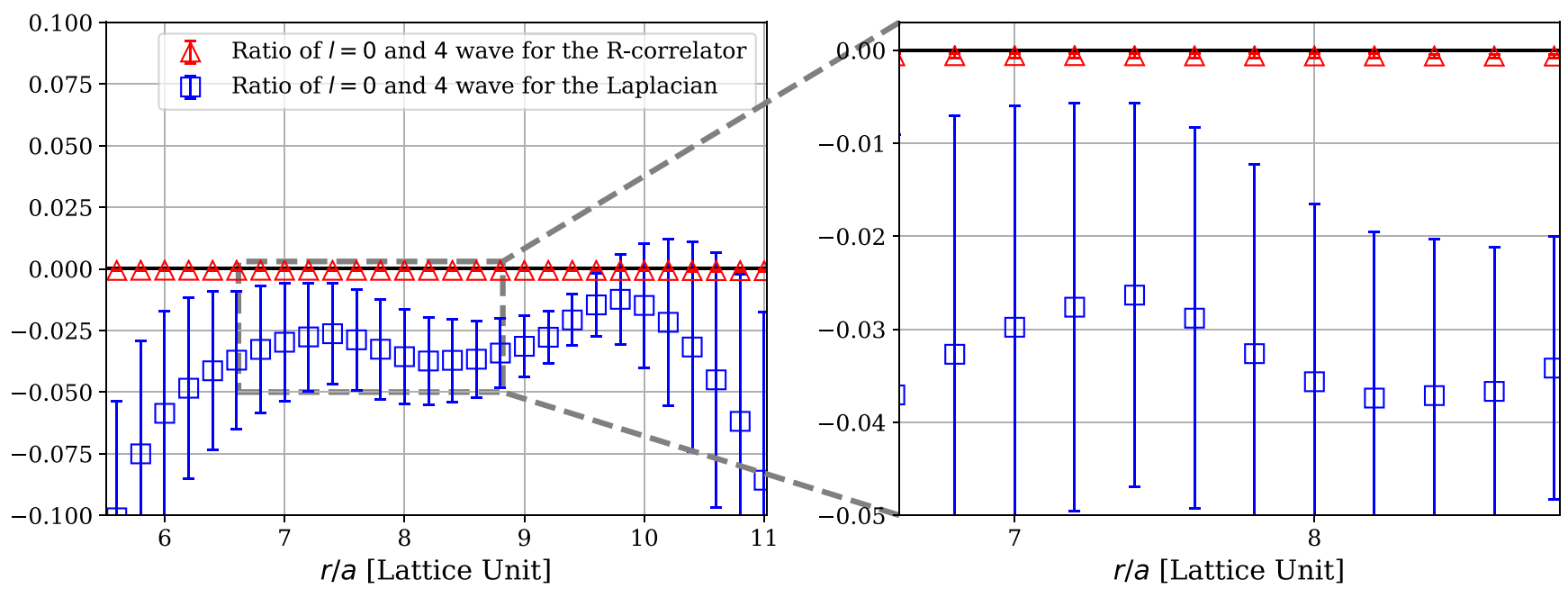

FIG. 4. Ratio of the spherical harmonics amplitudes, $g_{40}(r) / g_{00}(r)$, for the $R$ correlator (red triangles) and Laplacian term (blue squares). 


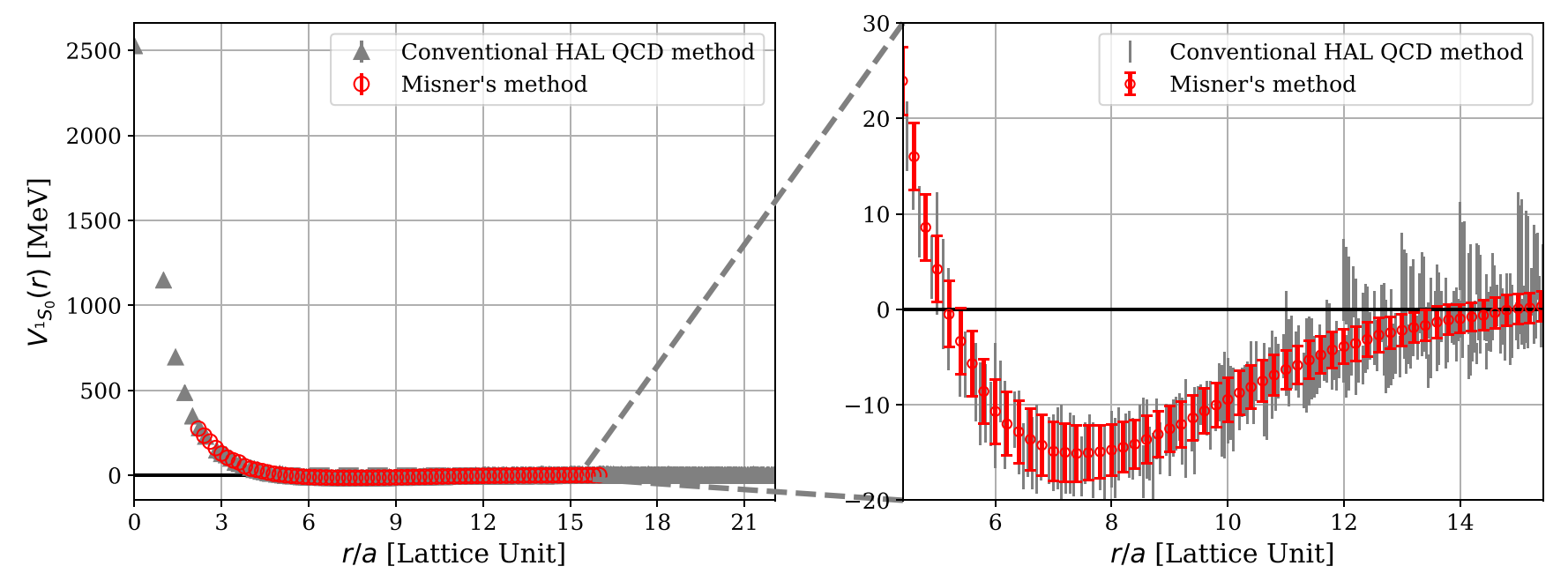

FIG. 5. The potential for the spin-singlet $\Lambda_{c} N$ system at $m_{\pi} \simeq 700 \mathrm{MeV}$, constructed using the time-dependent HAL QCD method from the $A_{1}^{+}$-projected $R$ correlator at $t-t_{0}=13 a$. The gray points show the potential from the conventional $A_{1}^{+}$projection, while the red points correspond to that from Misner's $S$-wave extraction.

term reveals that the $l=4$ component is indeed larger than the case of the $R$ correlator as shown in Fig. 4. The origin of these $l \geq 4$ components in the conventional Laplacian is most likely the $l \geq 4$ components in the $R$ correlator enhanced by the Laplacian, rather than the discretization error in the conventional Laplacian operator itself. In fact, in the case of Misner's method, the difference between the results with the conventional Laplacian operator applied to $g_{00}(r)$ and those with the analytic Laplacian operator is found to be marginal.

In Fig. 5 we show the HAL QCD potentials for the spinsinglet $\Lambda_{c} N$ system from Misner's $S$-wave extraction (red points) and the conventional $A_{1}^{+}$projection (gray points). In the case of Misner's extraction, the potential is found to be free from comb-like structures. In the case of the conventional projection, however, the potential has large comblike structures. The main origin is the $l \geq 4$ components in the Laplacian term for the potential, which are enhanced by applying the Laplacian to the $R$ correlator, even though $l \geq 4$ components are small in the $R$ correlator itself.

\section{Parameter dependencies for potentials in Misner's method}

Here we discuss the dependencies of the potentials in Misner's method on the parameters $\Delta, n_{\max }$ and $l_{\max }$, which correspond to the thickness of the spherical shell, and the maximum number of bases for the radial function and spherical harmonics, respectively. Throughout this section, the potential is constructed from the $l=0$ component of the NBS wave function.

We first show the $n_{\max }$ dependence with the other parameters fixed to $\Delta=a$ and $l_{\max }=4$ in the top two panels of Fig. 6, where we vary the value of $n_{\max }$ from 1 to 5. In Fig. 6 we plot only the central values of the potentials without statistical errors in order to make it easier to see the dependence on the parameters in Misner's method. Note that the magnitudes of the statistical errors are found to be stable against changing parameters. For $n_{\max }=1$, the potential does not reproduce the correct behavior. The reason is that the second derivative of the Legendre polynomial $P_{n}^{\prime \prime}(x)$ is zero for $n=0$ and 1 , and thus the Laplacian term in the potential from the spherical harmonics amplitude vanishes. The small contribution to the potential at $n_{\max }=1$ comes from the time-derivative terms [the first and second terms in Eq. (10)]. Thus, it is necessary to take $n_{\max } \geq 2$, for which we find that the potentials are almost stable against changes in $n_{\max }$. While we observe small oscillations of the potential for $n_{\max } \geq 4$, they are probably due to the numerical instabilities associated with small eigenvalues of $\mathcal{G}_{A B}$ caused by a large $n_{\max }$, as discussed in Sec. III C. Recall also that the discretization errors of the radial orthonormal basis function $G_{n}^{R, \Delta}(r)$ are known to be $\mathcal{O}\left(\Delta^{n_{\max }+2}\right)$ [19]. Since the discretization errors in our lattice QCD action [20] are $\mathcal{O}\left(a^{2}\right)$, a choice $n_{\max }=2$ with $\Delta=\mathcal{O}(a)$ is reasonable.

We next present the $\Delta$ dependence of the potential in the middle two panels of Fig. 6, where we take $\Delta=0.2 a, 0.5 a, a, 1.5 a$, and $2 a$ with $n_{\max }=2$ and $l_{\max }=4$ fixed. For $\Delta=0.2 a$ and $0.5 a$, we find the comb-like structures even in the potential constructed from the $l=0$ component, while such structures are absent for the potential with $\Delta \geq a$. This can be understood from the fact that the number of data points in the spherical shell becomes too small for small $\Delta$ to accurately reproduce the spherical harmonics amplitude. For larger $\Delta$, on the other hand, there appear small deviations from the potential with $\Delta=a$ at short distances, while the potentials at long distances are stable against changes in $\Delta$. This may be explained by the fact that variations of the $R$ correlator in the spherical shell become sizable for larger $\Delta$, so we need 

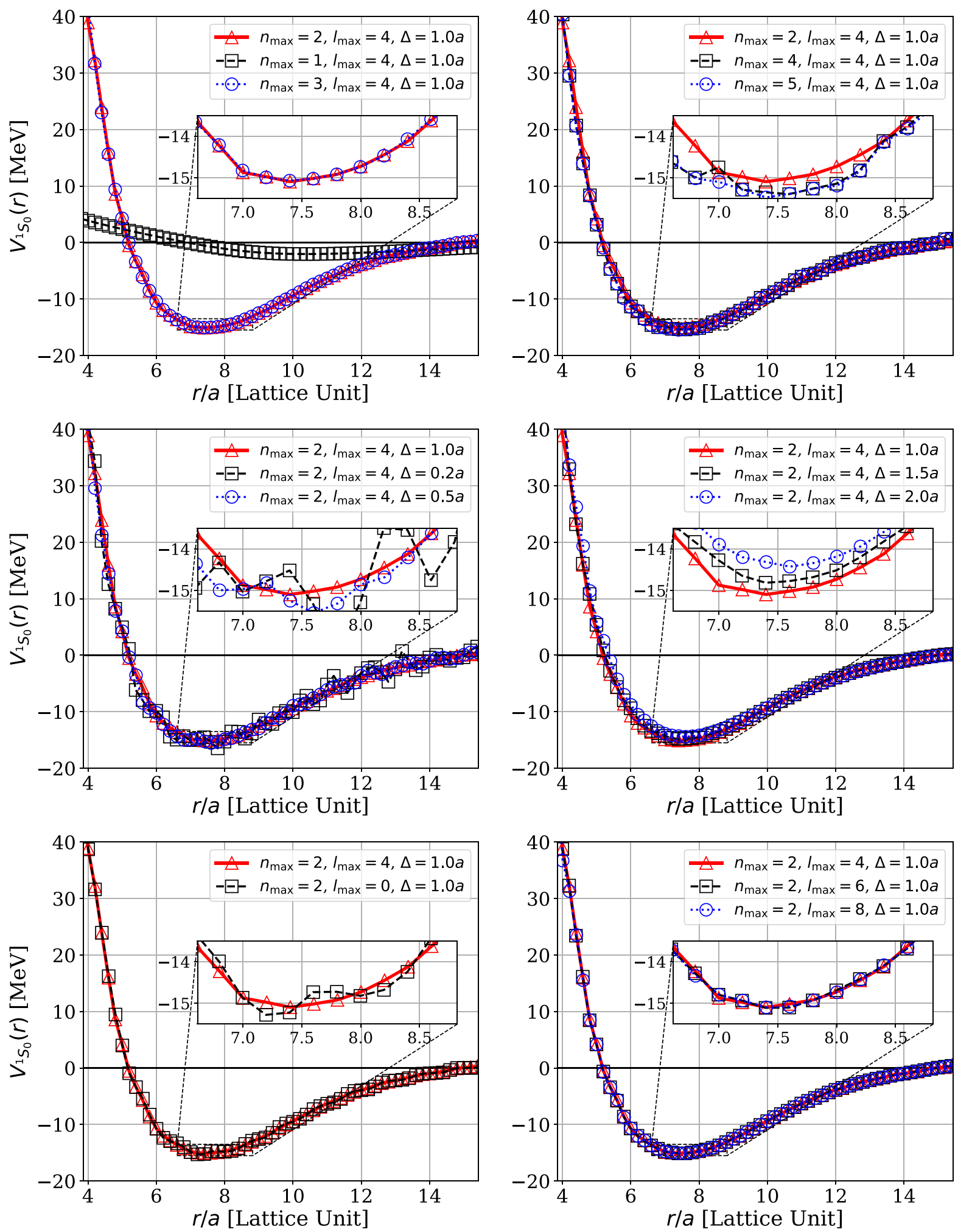

FIG. 6. The parameter dependence in Misner's method for the potential. The red line (with triangles) shows the HAL QCD potential constructed with Misner's method with the parameters $n_{\max }=2, l_{\max }=4$, and $\Delta=a$, while the other lines (with other symbols) represent those with the parameters specified in the legends.

to enlarge $n_{\max }$ accordingly to approximate the spherical harmonics amplitudes precisely. In fact, by taking larger values of $n_{\max }$ in the case of $\Delta=1.5 a, 2 a$, we find that the results tend to converge to that with $\Delta=a$ and $n_{\max }=2$. From these observations, we take $\Delta=a$ and $n_{\max }=2$ in this paper. 

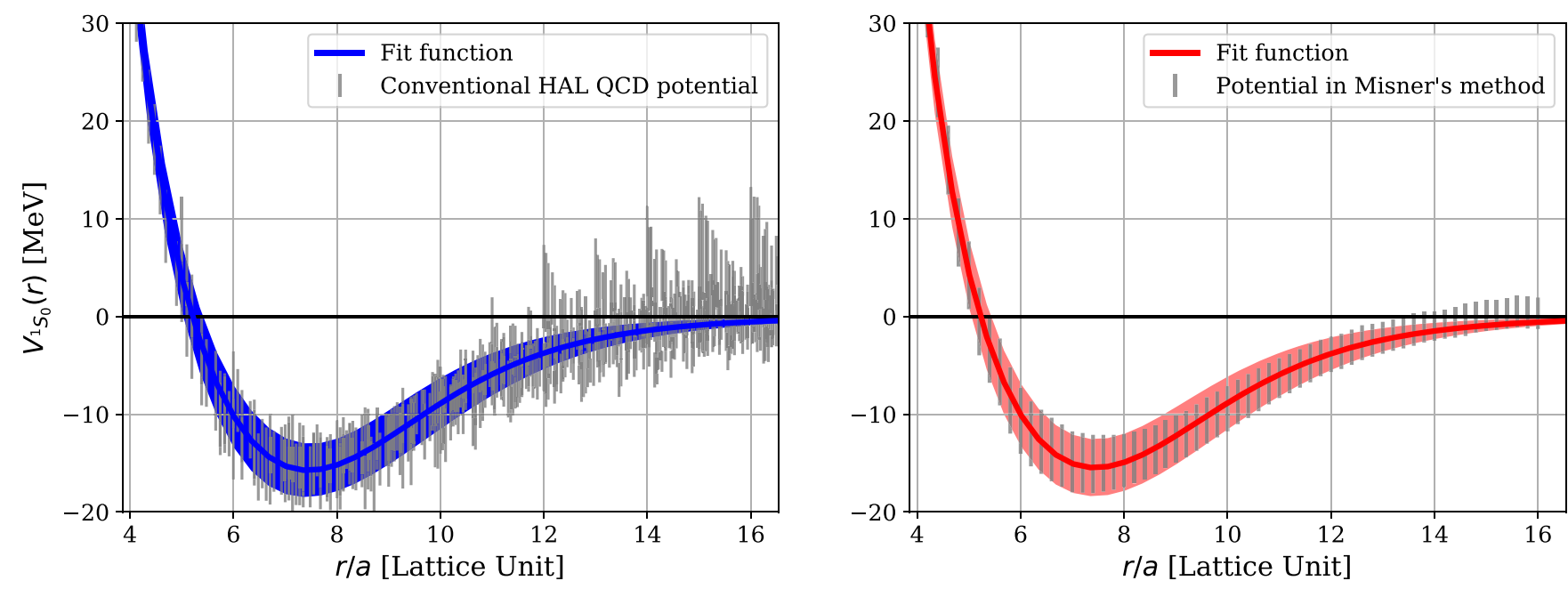

FIG. 7. Fits for the HAL QCD potential obtained from the conventional $A_{1}^{+}$projection (left) and from Misner's $S$-wave extraction (right). The fit range is $r \in[2 a, 16 a]$ for both cases.

Finally, we investigate the $l_{\max }$ dependence with $\Delta$ and $n_{\max }$ fixed, as shown in the lower two panels of Fig. 6. We take $l_{\max }=0,4,6$, and 8 , while keeping $\Delta=a$ and $n_{\max }=2$. The potentials are stable against changes in $l_{\max }$. While the potential is rather reasonable even in the case of $l_{\max }=0$, small oscillations are observed in the potential for this case. Such oscillations are absent for $l_{\max } \geq 4$, indicating that the $l_{\max }=4$ component has small but non-negligible contributions in the $R$ correlator, while $l_{\max }>4$ components are sufficiently small. The $l=6$ component in the $R$ correlator obtained with $l_{\max } \geq 6$ is actually found to be negligible. Therefore, we take $l_{\max }=4$ in this paper as a conservative choice to avoid numerical instabilities for larger $l_{\max }$.

\section{PHASE SHIFTS FOR THE SPIN-SINGLET $\Lambda_{c} N$ SYSTEM}

Here we compare the scattering phase shifts calculated from the HAL QCD potential obtained by Misner's $S$-wave extraction with those obtained by the conventional $A_{1}^{+}$ projection, in order to estimate the effects of $l \geq 4$ partial waves on physical observables. For this purpose, we fit both potentials using the fit function

$$
V_{\mathrm{fit}}(r)=a_{1} e^{-\left(\frac{r}{a_{2}}\right)^{2}}+a_{3}\left[\left(1-e^{-a_{4} r^{2}}\right) \frac{e^{-a_{5} r}}{r}\right]^{2},
$$

with the fitting range $r \in[2 a, 16 a]$, where both the fit and original data are shown in Fig. 7. While the conventional HAL QCD potential has large comb-like structures, the fit parameters $a_{i}$ are almost identical for both potentials, and, more surprisingly, the magnitudes of statistical errors are also found to be similar. This observation indicates that $l \geq 4$ contributions in the conventional HAL QCD potential hardly affect the fit of the potential. The agreement for the fit parameters between the two cases is most likely due to the fact that the fit function for the potential is taken to be isotropic [see Eq. (39)]. In the fit, we employ the uncorrelated fit; a more systematic study with the correlated fit is left for future studies.

By solving the Schrödinger equation numerically with the fitted potentials, we extract the scattering phase shifts, which are shown in Fig. 8. As expected from the fit results for the potentials, both the central values of the scattering phase shifts and their statistical errors are almost identical for the two methods.

In the analysis with the conventional HAL QCD method, while the potential is affected by contaminations from

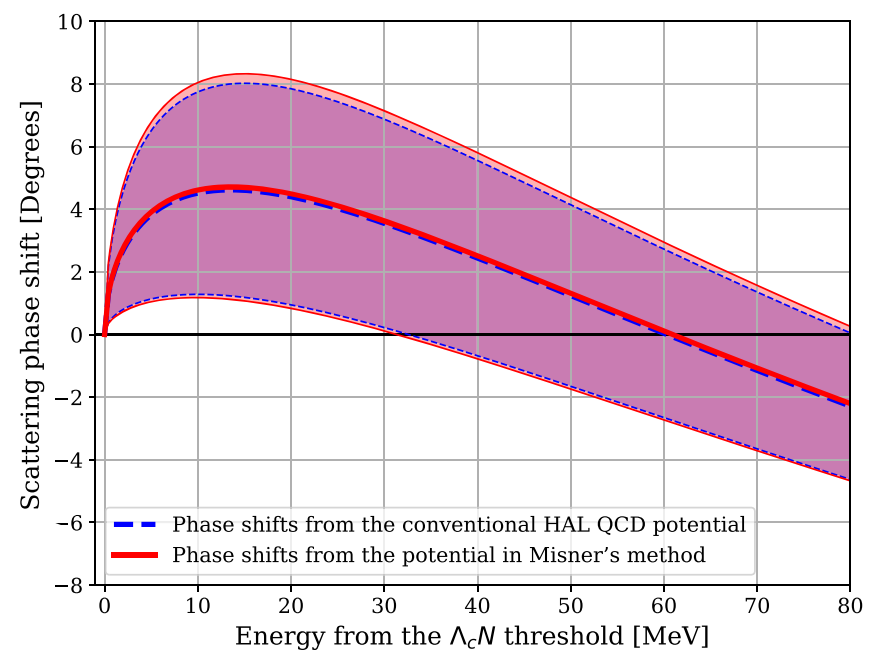

FIG. 8. Scattering phase shifts for the spin-singlet $\Lambda_{c} N$ system. The blue dashed line shows the phase shifts calculated from the potential by the conventional $A_{1}^{+}$projection, while the red solid line represents the phase shifts calculated from the potential by Misner's $S$-wave extraction. 
higher partial waves with $l \geq 4$, it is confirmed that the results for the scattering phase shifts are not affected by such systematics for both central values and magnitudes of errors. The conventional HAL QCD potential sometimes shows large fluctuations, in particular for $N N$ channels at lighter pion masses. The results in this paper tell us that these fluctuations are mainly systematic and are due to contaminations of higher partial waves enhanced by the Laplacian. By removing higher partial-wave components from the $R$ correlator and calculating the Laplacian analytically, the analysis with Misner's method reveals genuine statistical errors of the potential. The agreement between errors of the scattering phase shifts (or equivalently the fitted potential) in the two analyses with the conventional method and Misner's method provides a valuable check of the results.

\section{SUMMARY AND CONCLUSION}

In this paper, we have performed the approximated partial-wave decomposition by Misner's method to lattice QCD data in order to extract the $l=0$ component of the $A_{1}^{+}$-projected NBS wave functions and its Laplacian for the $\Lambda_{c} N$ system in the spin-singlet channel, calculated in the $(2+1)$-flavor QCD on $(32 a \mathrm{fm})^{3} \times(64 a \mathrm{fm})$ at $m_{\pi} \simeq$ $700 \mathrm{MeV}$ [20].

We obtained the following results. While the $A_{1}^{+}$projected NBS wave functions contain small contaminations from $l \geq 4$ partial waves, such contaminations are enhanced if the Laplacian approximated by the secondorder difference is applied to the NBS wave function, which cause large fluctuations in the conventional HAL QCD potential. With the use of Misner's method, since the Laplacian can be calculated analytically for the designated $(l=0)$ partial-wave component in the NBS wave function, the potential is free from contaminations from higher partial waves, and thus its fluctuations become much smaller. Therefore, Misner's method is very useful for reducing superficial fluctuations of the potential. If we fit the potentials as a function of $r$, both the central values and statistical errors of the fit parameters are almost independent of whether the conventional HAL QCD potential or the potential extracted with Misner's method is used as input. Consequently, the scattering phase shifts agree between the two methods. This agreement demonstrates not only that Misner's method works well in the HAL QCD method, but also that the contaminations from higher partial waves in the study of $S$-wave scatterings are well under control even in the conventional HAL QCD method.

Since one can approximately obtain the spherical harmonics amplitude for an arbitrary $l$ component by Misner's method, it is interesting to apply the method to extract the potentials from higher partial-wave channels. For example, in order to extract the tensor potential, one needs to obtain the $l=0$ and $l=2$ components of the NBS wave function separately. In the conventional HAL QCD method, one extracts the $l=2$ component from the NBS wave function by the projection $\left(1-P^{A_{1}^{+}}\right)$, which however also contains $l \geq 4$ components. By employing Misner's method, on the other hand, one can extract the $l=0$ and $l=2$ components separately without contaminations from higher partial waves, and thus obtain the tensor potential as well as the central potential without comb-like structures.

\section{ACKNOWLEDGMENTS}

Numerical data used in this study were obtained by the KEK supercomputer system (BG/Q) [Project number: 14/15-21, 15/16-12]. This work is supported in part by JSPS Grant-in-Aid for Scientific Research Grants No. JP19K03879, JP18H05236, JP18H05407, JP16H03978, and JP15K17667, by a priority issue (Elucidation of the fundamental laws and evolution of the universe) to be tackled by using the Post "K" Computer, and the by Joint Institute for Computational Fundamental Science (JICFuS). The authors thank all the members of the HAL QCD Collaboration for discussion.

\section{APPENDIX: APPROXIMATED PARTIAL-WAVE DECOMPOSITION AT FIXED $r$}

In this appendix, we propose a simpler method to extract $g_{00}(r)$ from the discrete data, which is compared with Misner's method. Here we consider the $A_{1}^{+}$-projected NBS wave function defined by

$$
\begin{aligned}
\psi^{A_{1}^{+}}(\vec{x}) & \equiv P^{A_{1}^{+}} \psi(\vec{x}) \\
& =Y_{00}^{A_{1}^{+}}(\theta, \phi) g_{00}(r)+\sum_{m=0, \pm 4} Y_{4 m}^{A_{1}^{+}}(\theta, \phi) g_{4 m}(r)+\cdots
\end{aligned}
$$

where $P^{A_{1}^{+}}$is the projection operator to the $A_{1}^{+}$representation, $Y_{00}^{A_{1}^{+}}(\theta, \phi)$ and $Y_{4 m}^{A_{1}^{+}}(\theta, \phi)$ stand for the $A_{1}^{+}$-projected spherical harmonics, given by

$$
\begin{gathered}
Y_{00}^{A_{1}^{+}}(x, y, z)=Y_{00}(x, y, z)=\frac{1}{\sqrt{4 \pi}}, \\
Y_{40}^{A_{1}^{+}}(x, y, z)=\frac{7}{8 \sqrt{\pi}} \frac{x^{4}+y^{4}+z^{4}-3\left(x^{2} y^{2}+y^{2} z^{2}+z^{2} x^{2}\right)}{r^{4}}, \\
Y_{4,+4}^{A_{1}^{+}}(x, y, z)=Y_{4,-4}^{A_{1}^{+}}(x, y, z)=\sqrt{\frac{5}{14}} Y_{40}^{A_{1}^{+}}(x, y, z),
\end{gathered}
$$

and the ellipsis denotes higher angular momentum components such as $l=6,8, \ldots$ In the $A_{1}^{+}$representation, the $l=4$ components become nonzero only for $m=0, \pm 4$. Since $Y_{40}^{A_{1}^{+}}$(and $Y_{4, \pm 4}^{A_{1}^{+}}$) has an angular dependence, the NBS 

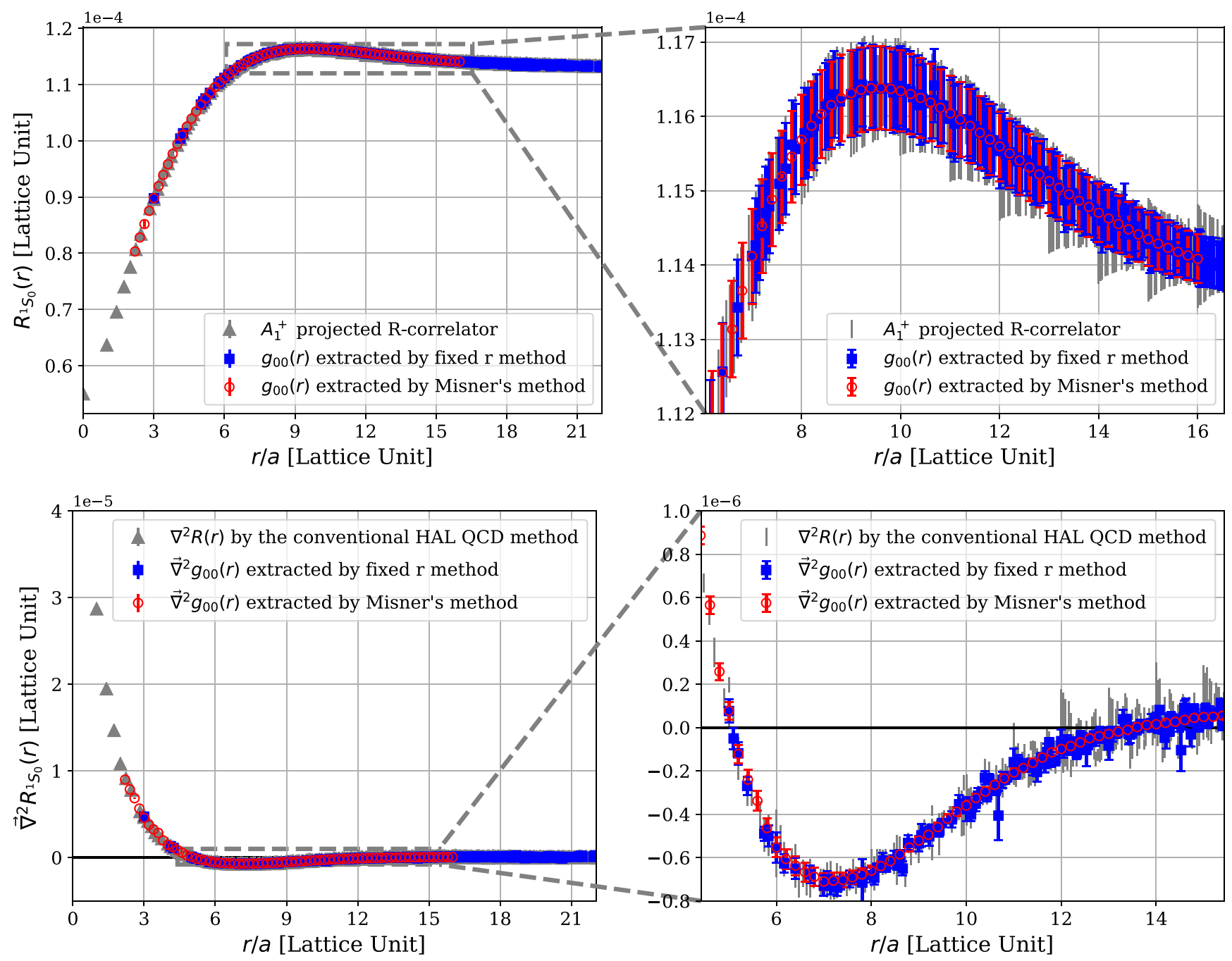

FIG. 9. The $R$ correlator for the spin-singlet $\Lambda_{c} N$ system at $m_{\pi} \simeq 700 \mathrm{MeV}$ (upper panels) and its Laplacian term (lower panels). The $R$ correlator is calculated at $t-t_{0}=13 a$. The spherical harmonics amplitude $g_{00}(r)$ and its Laplacian term extracted using the method in this appendix are plotted (blue points), together with those from Misner's method (red points) as well as the original $A_{1}^{+}$-projected $R$ correlator and its Laplacian term (gray points).

wave function at given $r$ is multivalued, which gives rise to comb-like structures in the potential in the radial coordinate.

Let us assume that there are $N$ points $\vec{x}_{i}(i=1, \ldots, N)$ that satisfy $\left|\vec{x}_{i}\right|=r$ but cannot be transformed into each other by a cubic rotation. Neglecting components with $l \geq 6$, we have

$$
\begin{aligned}
\psi^{A_{1}^{+}}\left(\vec{x}_{i}\right) & \simeq Y_{00}^{A_{1}^{+}} g_{00}(r)+\sum_{m=0, \pm 4} Y_{4 m}^{A_{1}^{+}}\left(\vec{x}_{i}\right) g_{4 m}(r) \\
& =Y_{00}^{A_{1}^{+}} g_{00}(r)+Y_{40}^{A_{1}^{+}}\left(\vec{x}_{i}\right) g_{4}(r),
\end{aligned}
$$

where we omit the arguments for the constant $Y_{00}^{A_{1}^{+}}$, and $g_{4}(r) \equiv g_{40}(r)+\sqrt{\frac{5}{14}}\left(g_{44}(r)+g_{4-4}(r)\right)$. Equation (A5) can be compactly written as

$$
\left(\begin{array}{c}
\psi^{A_{1}^{+}}\left(\vec{x}_{1}\right) \\
\vdots \\
\psi^{A_{1}^{+}}\left(\vec{x}_{N}\right)
\end{array}\right)=\left(\begin{array}{cc}
Y_{00}^{A_{1}^{+}} & Y_{40}^{A_{1}^{+}}\left(\vec{x}_{1}\right) \\
\vdots & \vdots \\
Y_{00}^{A_{1}^{+}} & Y_{40}^{A_{1}^{+}\left(\vec{x}_{N}\right)}
\end{array}\right)\left(\begin{array}{c}
g_{00}(r) \\
g_{4}(r)
\end{array}\right),
$$

where the matrix on the right-hand side is (in general) a $N \times 2$ rectangular matrix. If $N>2$, we solve Eq. (A6) using the singular-value decomposition (SVD) for the rectangular matrix. The SVD for a $M \times N(M>N)$ rectangular matrix $A$ is denoted as $A=U \Sigma V^{\dagger}$, where $U, V$ are unitary matrices and $\Sigma$ is a diagonal matrix of the singular values. Then, the generalized inverse matrix is defined as $A^{-1}=V \Sigma^{-1} U^{\dagger}$, where $\Sigma$ and $\Sigma^{-1}$ are given by 


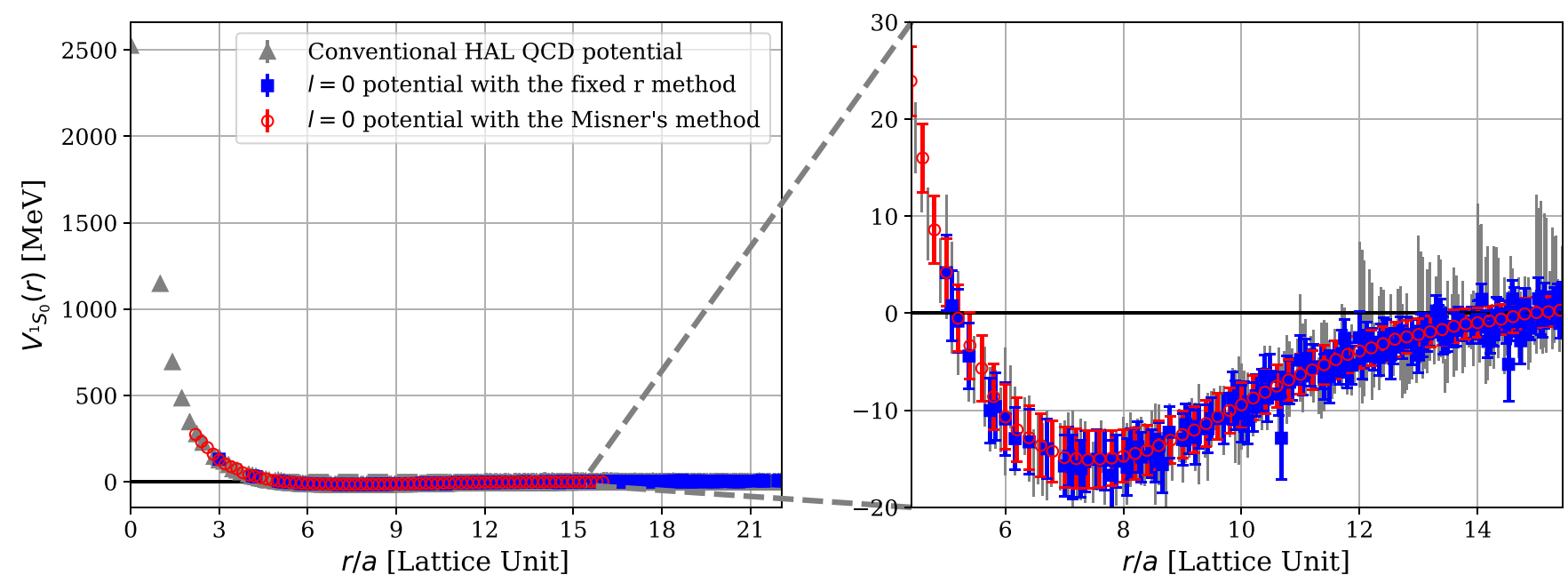

FIG. 10. The potential for the spin-singlet $\Lambda_{c} N$ system. The potential is constructed using the time-dependent HAL QCD method from the $A_{1}^{+}$-projected $R$ correlator calculated at $t-t_{0}=13 a$ at $m_{\pi} \simeq 700 \mathrm{MeV}$. The gray points show the potential calculated using the conventional HAL QCD method, while the blue and red points correspond to the potential constructed from the spherical harmonics amplitude $g_{00}(r)$ calculated using the method in this appendix and Misner's method, respectively.

$$
\begin{gathered}
\Sigma \equiv\left(\begin{array}{c}
\operatorname{diag}\left(\sigma_{1}, \ldots, \sigma_{N}\right) \\
\mathbf{0}_{(M-N) \times N}
\end{array}\right), \\
\Sigma^{-1} \equiv\left(\operatorname{diag}\left(\sigma_{1}^{-1}, \ldots, \sigma_{N}^{-1}\right) \quad \mathbf{0}_{N \times(M-N)}\right),
\end{gathered}
$$

where $\mathbf{0}_{M \times N}$ represents the $M \times N$ zero matrix. Here we assume that all singular values are nonzero; otherwise, the generalized inverse matrix cannot be defined.

An extension to higher angular momentum components than $l=4$ is straightforward. Including the $l=6$ component, for instance, we can extract the radial functions for $l=0,4$, and 6 by solving the equation

$$
\left(\begin{array}{c}
\psi^{A_{1}^{+}}\left(\vec{x}_{1}\right) \\
\vdots \\
\psi^{A_{1}^{+}}\left(\vec{x}_{N}\right)
\end{array}\right)=\left(\begin{array}{ccc}
Y_{00}^{A_{1}^{+}} & Y_{40}^{A_{1}^{+}}\left(\vec{x}_{1}\right) & Y_{60}^{A_{1}^{+}}\left(\vec{x}_{1}\right) \\
\vdots & \vdots & \vdots \\
Y_{00}^{A_{1}^{+}} & Y_{40}^{A_{1}^{+}}\left(\vec{x}_{N}\right) & Y_{60}^{A_{1}^{+}}\left(\vec{x}_{N}\right)
\end{array}\right)\left(\begin{array}{c}
g_{00}(r) \\
g_{4}(r) \\
g_{6}(r)
\end{array}\right) \text {. }
$$

Since the matrix on the right-hand side is a $N \times 3$ rectangular matrix, we need at least three points that satisfy $\left|\vec{x}_{i}\right|=r$ but are not transformed by the cubic rotation.

We next consider the extraction of the Laplacian for a radial function such as $g_{00}(r)$ in order to construct the potentials. Neglecting components with $l \geq 6$ again, the Laplacian of the NBS wave function in Eq. (A5) becomes

$$
\vec{\nabla}^{2} \psi^{A_{1}^{+}}\left(\vec{x}_{i}\right)=Y_{00}^{A_{1}^{+}} \vec{\nabla}^{2} g_{00}(r)+\vec{\nabla}^{2}\left[Y_{40}^{A_{1}^{+}}\left(\vec{x}_{i}\right) g_{4}(r)\right],
$$

where the second term is evaluated in the continuum relation as
$\vec{\nabla}^{2}\left[Y_{4}^{A_{1}^{+}}\left(\vec{x}_{i}\right) g_{4}(r)\right]=Y_{4}^{A_{1}^{+}}\left(\vec{x}_{i}\right)\left[\vec{\nabla}^{2}-\frac{4(4+1)}{r^{2}}\right] g_{4}(r)$

Combining Eqs. (A5) with (A10), we have

$$
\begin{aligned}
& \left(\begin{array}{c}
\psi^{A_{1}^{+}}\left(\vec{x}_{1}\right) \\
\vec{\nabla}^{2} \psi^{A_{1}^{+}\left(\vec{x}_{1}\right)} \\
\vdots \\
\psi^{A_{1}^{+}\left(\vec{x}_{N}\right)} \\
\vec{\nabla}^{2} \psi^{A_{1}^{+}}\left(\vec{x}_{N}\right)
\end{array}\right)=\left(\begin{array}{cccc}
Y_{00}^{A_{1}^{+}} & 0 & Y_{40}^{A_{1}^{+}}\left(\vec{x}_{1}\right) & 0 \\
0 & Y_{00}^{A_{1}^{+}} & -\frac{4(4+1)}{r^{2}} & Y_{40}^{A_{1}^{+}}\left(\vec{x}_{1}\right) \\
\vdots & \vdots & \vdots & \vdots \\
Y_{00}^{A_{1}^{+}} & 0 & Y_{40}^{A_{1}^{+}}\left(\vec{x}_{N}\right) & 0 \\
0 & Y_{00}^{A_{1}^{+}} & -\frac{4(4+1)}{r^{2}} & Y_{40}^{A_{1}^{+}}\left(\vec{x}_{N}\right)
\end{array}\right) \\
& \times\left(\begin{array}{c}
g_{00}(r) \\
\vec{\nabla}^{2} g_{00}(r) \\
g_{4}(r) \\
\vec{\nabla}^{2} g_{4}(r)
\end{array}\right),
\end{aligned}
$$

which can be solved using SVD. Note that the Laplacian on the left-hand-side of Eq. (A10) is approximated by the second-order difference, which has $\mathcal{O}\left(a^{2}\right)$ discretized errors.

An advantage of this method over Misner's method is that we do not need a parameter to extract the $l=0$ component of the NBS wave function. Applying this method, we extract the $l=0$ component of the $A_{1}^{+}$projected $R$ correlator for the spin-singlet $\Lambda_{c} N$ system. In Fig. 9, $g_{00}(r)$ and its Laplacian are compared with those extracted by Misner's method. The spherical harmonic amplitude $g_{00}(r)$ extracted by this method does not show 
comb-like structures and is consistent with $g_{00}(r)$ in Misner's method, while its Laplacian has comb-like structures, which probably originate from discretized errors due to the Laplacian on the left-hand-side of Eq. (A10). Consequently, the potential constructed from the $l=0$ component using this method (shown in Fig. 10) also has comb-like structures. Therefore, Misner's method works better for the HAL QCD potential than the method in this section, which however may be used to extract $g_{00}(r)$ only [21].
[1] M. Lüscher, Nucl. Phys. B354, 531 (1991).

[2] K. Murano, N. Ishii, S. Aoki, and T. Hatsuda, Prog. Theor. Phys. 125, 1225 (2011).

[3] Charles W. Misner, Classical Quantum Gravity 21, S243 (2004).

[4] D. R. Fiske, J. G. Baker, J. R. van Meter, D. Choi, and J. M. Centrella, Phys. Rev. D 71, 104036 (2005).

[5] N. Ishii, S. Aoki, and T. Hatsuda, Phys. Rev. Lett. 99, 022001 (2007).

[6] S. Aoki, T. Hatsuda, and N. Ishii, Prog. Theor. Phys. 123, 89 (2010).

[7] N. Ishii, S. Aoki, T. Doi, T. Hatsuda, Y. Ikeda, T. Inoue, K. Murano, H. Nemura, and K. Sasaki (HAL QCD Collaboration), Phys. Lett. B 712, 437 (2012).

[8] S. Aoki et al. (HAL QCD Collaboration), Prog. Theor. Exp. Phys. (2012), 01A105.

[9] T. Inoue, N. Ishii, S. Aoki, T. Doi, T. Hatsuda, Y. Ikeda, K. Murano, H. Nemura, and K. Sasaki (HAL QCD Collaboration), Prog. Theor. Phys. 124, 591 (2010).

[10] S. Aoki, N. Ishii, T. Doi, Y. Ikeda, and T. Inoue, Phys. Rev. D 88, 014036 (2013).

[11] S. Gongyo and S. Aoki, Prog. Theor. Exp. Phys. (2018), 093 B03.

[12] T. Iritani et al. (HAL QCD Collaboration), J. High Energy Phys. 10 (2016) 101.
[13] T. Iritani, S. Aoki, T. Doi, T. Hatsuda, Y. Ikeda, T. Inoue, N. Ishii, H. Nemura, and K. Sasaki (HAL QCD Collaboration), Phys. Rev. D 96, 034521 (2017).

[14] T. Iritani, S. Aoki, T. Doi, T. Hatsuda, Y. Ikeda, T. Inoue, N. Ishii, H. Nemura, and K. Sasaki (HAL QCD Collaboration), J. High Energy Phys. 03 (2019) 007.

[15] T. Iritani, S. Aoki, T. Doi, S. Gongyo, T. Hatsuda, Y. Ikeda, T. Inoue, N. Ishii, H. Nemura, and K. Sasaki (HAL QCD Collaboration), Phys. Rev. D 99, 014514 (2019).

[16] S. Okubo and R. E. Marshak, Ann. Phys. (N.Y.) 4, 166 (1958).

[17] M. E. Rupright, arXiv:gr-qc/0606127.

[18] If $N_{R, \Delta}-M<0$ (and there is no symmetry), $\mathcal{G}_{A B}$ has zero eigenvalues.

[19] D. R. Fiske, Classical Quantum Gravity 23, 5951 (2006).

[20] T. Miyamoto et al. (HAL QCD Collaboration), Nucl. Phys. A971, 113 (2018).

[21] On this point, the method in this section essentially corresponds to Misner's method with the thickness of the spherical shell $\Delta \rightarrow 0$. With the generalization of $\Delta \neq 0$, Misner's method can utilize more data points and thus obtain $g_{00}(r)$ at more points of $r$ (red) than the method in this section (blue) at short distances. 